\title{
Comparison of Picard Groups in Dimension 1
}

\author{
Henri Lombardi $\left(^{*}\right)$, Claude Quitté $\left(^{\dagger}\right)$
}

September 10, 2007

\begin{abstract}
We compare two Picard groups in dimension one. Our proofs are constructive and the results generalize a theorem of J. Sands [11].
\end{abstract}

MSC 2000: 13C15, 13C20, 03F65, 13F45

Keywords: Krull Dimension, Picard Group, Constructive Mathematics.

\section{Introduction}

In [11] J. Sands generalizes a theorem of Siegel [13]. He explains the link between the Picard group of the integer ring of a number field and that of an order of this number field. He applies his results to computing bounds on regulators.

We give here an extension of Sands' results in the case of two integral domains $A \subseteq B$ of dimension 1 when $B$ is finite over $A$ with the same fraction field. Our proof is constructive and gives a possible algorithm for Computer Algebra (e.g., for computing the Picard group of algebraic curves) when hypotheses are satisfied in an explicit way.

Let us recall that the conductor c $(A, B)$ of $A$ in $B$ (when $A$ is a subring of $B$ ) is an ideal of $A$ and $B$ defined by:

$$
\mathrm{c}(A, B)=\{x \in A \mid x B \subseteq A\} .
$$

The fact that our theorem replaces the conductor of $A$ in $B$ by a nonzero ideal contained in the conductor, improves, w.r.t. the original theorem, the possibility of a concrete computation.

In the first section, we give partial results in the case of an arbitrary extension of rings. This gives as a particular case a construction of Schanuel related to seminormality. The second section is devoted to reminders concerning the constructive approach of Krull dimension. Section 3 gives the proof of the main theorem of this paper:

Theorem 7 Let $A \subseteq B$ be two integral domains of dimension 1 with the same fraction field and such that $B$ is a finitely generated $A$-module. If $\mathfrak{f}$ is a nonzero ideal of $B$ contained in the conductor of $A$ in $B$, then we have the following exact sequence:

$$
1 \longrightarrow U(B) / U(A) \stackrel{i[b] \mapsto[b]}{\longrightarrow} U(B / \mathfrak{f}) / U(A / \mathfrak{f}) \stackrel{j[b] \mapsto[b A+\mathfrak{f}]}{\longrightarrow} \operatorname{Pic}(A) \stackrel{\pi[\mathfrak{a}] \mapsto[\mathfrak{a} B]}{\longrightarrow} \operatorname{Pic}(B) \longrightarrow 1
$$

\footnotetext{
* Laboratoire de Mathématiques, CNRS UMR 6623, UFR des Sciences et Techniques, Université de Franche-Comté, 25030 Besançon cedex, FRANCE, email: henri.lombardi@univ-fcomte.fr

† Laboratoire de Mathématiques, CNRS UMR 6086, SP2MI, Boulevard 3, Téléport 2, BP 179, 86960 Futuroscope Cedex, FRANCE, email: quitte@mathlabo.univ-poitiers.fr
} 
Let us recall that this theorem was given by Dedekind [5] in algebraic number theory when $B$ is the ring of all integers in a number field.

Our proofs are given in the usual style of constructive algebra (cf. [9]). We have no Notherian hypothesis. We use a simple characterization "without prime ideals" of Krull dimension ([2]): this characterization has already lead to elementary and constructive proofs for some classical results in commutative algebra that were previously purely ideal theorems (cf. [3, 4, 6, 8]).

Our theorem is new from two points of view. First we don't use any Nœtherian hypothesis, second we obtain an algorithm that makes explicit the exactness of the sequence.

Let us insist on the fact that theorems whose hypotheses mention the Krull dimension can have an algorithmic version only once Krull dimension has became an explicit notion.

Acknowledgments: We are pleased to thank the referee for his careful reading and relevant comments.

\section{Exact sequences for groups of invertible modules}

We note $U(C)$ the group of invertible elements of an arbitrary ring $C$.

Let us consider two commutative rings $A \subseteq B$. We say that a sub- $A$-module $M$ of $B$ is invertible if there exists a sub- $A$-module $N$ of $B$ such that $M . N=A$. This definition coincides with that of Bourbaki [1] when $B$ is the localization of $A$ at a multiplicative subset made up of non-zerodivisors. In this case we get for any sub- $A$-module $M^{\prime}$ of $B$ that $M . M^{\prime} \simeq M \otimes_{A} M^{\prime}$ via the canonical homomorphism. Indeed, we have $x_{1}, \ldots, x_{n}$ in $M, y_{1}, \ldots, y_{n}$ in $N$ such that $1=\sum_{i} x_{i} y_{i}$ and $x_{i} y_{j}$ in $A$. For any element $\sum_{k} z_{k} \otimes z_{k}^{\prime}$ in $M \otimes_{A} M^{\prime}$, since $y_{i} z_{k} \in N . M=A$, we have

$$
\sum_{k} z_{k} \otimes z_{k}^{\prime}=\sum_{k, i} x_{i} y_{i} z_{k} \otimes z_{k}^{\prime}=\sum_{k, i} x_{i}\left(y_{i} z_{k}\right) \otimes z_{k}^{\prime}=\sum_{k, i} x_{i} \otimes\left(y_{i} z_{k}\right) z_{k}^{\prime}=\sum_{i} x_{i} \otimes \sum_{k} y_{i} z_{k} z_{k}^{\prime},
$$

so the canonical surjection $M \otimes_{A} M^{\prime} \rightarrow M . M^{\prime}$ is injective. Thus invertible modules are rank 1 projective.

We note $\mathcal{M}_{\text {inv }}(A, B)$ the group of invertible sub- $A$-modules of $B$ and $\mathcal{I}_{\text {fr }}(B)$ the group of invertible fractionary ideals of $B$ : in other words $\mathcal{I}_{\text {fr }}(B)=\mathcal{M}_{\text {inv }}(B, \operatorname{Frac}(B))$, where $\operatorname{Frac}(B)$ is the total fraction ring of $B$. We note $\mathcal{P}_{\mathrm{fr}}(A, B)$ the sub-group of $\mathcal{M}_{\mathrm{inv}}(A, B)$ made up of monogeneous sub- $A$-modules of $B$. Finally $\operatorname{Pic}(A)$ is the group isomorphism classes of rank 1 projective modules.

\subsection{First exact sequence}

Let us consider an ideal $\mathfrak{f}$ of $B$ such that $\mathfrak{f} B \subseteq A$, i.e., $\mathfrak{f} \subseteq \mathrm{c}(A, B)$. We define a first exact sequence

$$
1 \longrightarrow U(A / \mathfrak{f}) \longrightarrow U(B / \mathfrak{f}) \stackrel{j}{\longrightarrow} \mathcal{M}_{\text {inv }}(A, B)
$$

- Since $\mathfrak{f}$ is an ideal of $A$ and $B$, one has $A / \mathfrak{f} \subseteq B / \mathfrak{f}$. So $U(A / \mathfrak{f}) \subseteq U(B / \mathfrak{f})$. This defines the (injective) left arrow.

- Let us look at the arrow $j$. For $b \in B$, let $j(b)=b A+\mathfrak{f}$. Up to now $j(b)$ is only a sub- $A$-module of $B$. Clearly $j(1)=A$, and $b_{1} \equiv b_{2}(\bmod \mathfrak{f})$ implies $j\left(b_{1}\right)=j\left(b_{2}\right)$. Remark that if $b_{1} \in B$ is invertible modulo $\mathfrak{f}$, then $b_{1} \mathfrak{f}+\mathfrak{f}^{2}=\mathfrak{f}$ (multiplying $b_{1} B+\mathfrak{f}=B$ by $\mathfrak{f}$ ). For all $b_{2} \in B$, we get $j\left(b_{1} b_{2}\right)=j\left(b_{1}\right) j\left(b_{2}\right)$ since

$$
j\left(b_{1}\right) j\left(b_{2}\right)=b_{1} b_{2} A+b_{1} \mathfrak{f}+b_{2} \mathfrak{f}+\mathfrak{f}^{2}=b_{1} b_{2} A+\left(b_{1} B+\mathfrak{f}\right) \mathfrak{f}+b_{2} \mathfrak{f}=b_{1} b_{2} A+\mathfrak{f}=j\left(b_{1} b_{2}\right) .
$$

This allows us to define the arrow $j: U(B / \mathfrak{f}) \mapsto \mathcal{M}_{\text {inv }}(A, B)$ that sends the class modulo $\mathfrak{f}$ of an (invertible modulo f) element $b \in B$ to $b A+\mathfrak{f}$ (this is an invertible $A$-module because $j$ is multiplicative). 
Remark that $j(b) B=B$ for all $b \in U(B / \mathfrak{f})$.

In order to show the exactness of the desired sequence, we have to compute ker $j$. Let $b$ be an element in $B$ invertible modulo $\mathfrak{f}$, such that $j(b)=A$, i.e., $\mathfrak{f}+b A=A$. Then $b A \subseteq A, b \in A$ and the equality $\mathfrak{f}+b A=A$ says that the element $b \in A$ is invertible modulo $\mathfrak{f}$.

In the sequel, when $b \in B$ we use without more precision the notations $\bar{b}, \widehat{b}, \widetilde{b}$ for the image of $b$ in a set $X$ through some natural map $B \rightarrow X$.

The previous result can be rephrased in the following way:

Proposition 1 The map $j: U(B / \mathfrak{f}) \rightarrow \mathcal{M}_{\text {inv }}(A, B)$ defined by $j(\bar{b})=b A+\mathfrak{f}$ induces an isomorphism from $U(B / \mathfrak{f}) / U(A / \mathfrak{f})$ onto some sub-group of $\mathcal{M}_{\text {inv }}(A, B)$ made up of invertible $A$-modules $\mathfrak{a}$ of $B$ such that $\mathfrak{a} B=B$.

\subsection{Second exact sequence}

We assume in the sequel that $B$ is integral over $A$.

The kernel of the homomorphism $U(B) \rightarrow U(B / \mathfrak{f}) / U(A / \mathfrak{f})$ is equal to $U(A)$ (an element of $A$ which is invertible in $B$ is invertible in $A$ ). This gives the arrow $i$ in "the second exact sequence":

$$
1 \longrightarrow U(B) / U(A) \stackrel{i}{\longrightarrow} U(B / \mathfrak{f}) / U(A / \mathfrak{f}) \stackrel{j}{\longrightarrow} \mathcal{M}_{\text {inv }}(A, B) / \mathcal{P}_{\mathrm{fr}}(A, B)
$$

Concerning the exactness it remains to compute ker $j$ (we keep the same name $j$ ). Let $b \in B$ be a unit modulo $\mathfrak{f}$ such that $j(b)$ is principal, i.e., $b A+\mathfrak{f}=b^{\prime} A$ with $b^{\prime} \in B$. Multiplying this equality by $B$, one obtains $b B+\mathfrak{f}=b^{\prime} B$, i.e., $B=b^{\prime} B$, and so $b^{\prime}$ is invertible in $B$. Multiplying $b A+\mathfrak{f}=b^{\prime} A$ by $b^{\prime-1}$ and letting $a=b b^{-1}$, one gets $a A+\mathfrak{f}=A$ so that $a \in A$ and is invertible modulo $\mathfrak{f}$. Finally $\bar{b}=i\left(\widehat{b^{\prime}}\right)$ as claimed.

The exactness of the above sequence may be rephrased in the following way.

Proposition 2 We assume that $B$ is integral over $A$. The map $j: U(B / \mathfrak{f}) / U(A / \mathfrak{f}) \rightarrow \mathcal{M}_{\mathrm{inv}}(A, B)$ defined by $j(\tilde{b})=b A+\mathfrak{f}$ induces an isomorphism of $U(B / \mathfrak{f}) / U(A / \mathfrak{f}) U(B)$ onto a sub-group of $\mathcal{M}_{\text {inv }}(A, B) / \mathcal{P}_{\text {fr }}(A, B)$. In other words, for an element $b \in B$ which is invertible modulo $\mathfrak{f}$, the sub- $A$ module $b A+\mathfrak{f}$ is principal if and only if there exists $u \in U(B)$ such that $u b \in A$.

Let us precise "in other words": let $b \in B$ be an element which is invertible modulo $\mathfrak{f}$. Its class is in $U(A / \mathfrak{f}) U(B)$ if and only if $b=a b^{\prime}(\bmod \mathfrak{f})$ with $a \in A$ invertible modulo $\mathfrak{f}$ and $b^{\prime} \in U(B)$. In this case we take $u=b^{\prime-1}$. Conversely, if such an $u$ exists, the element $a=u b$ is in $A$ and its class modulo $\mathfrak{f}$ is invertible in $B / \mathfrak{f}$, and thus invertible in $A / \mathfrak{f}$ since $B / \mathfrak{f}$ is integral over $A / \mathfrak{f}$.

\subsection{Application: seminormality}

Let $A \subseteq A[\alpha]$ with $\alpha^{2}$ and $\alpha^{3} \in A$. Let $B=A[\alpha]=A+\alpha A$ and $\mathfrak{f}=\alpha^{2} B=\alpha^{2} A+\alpha^{3} A$. For any $a \in A$ the element $b=1+a \alpha$ is invertible modulo $f$ (its inverse is $1-\alpha a$ ) and the sub- $A$-module $j(\bar{b})$ is invertible. By definition $j(\bar{b})=b A+\alpha^{2} A+\alpha^{3} A$ is also equal to $b A+\alpha^{2} A$ or $b A+\alpha^{3} A$ (since $\alpha^{3}=\alpha^{3} b-\left(a \alpha^{2}\right) \alpha^{2}$ and $\left.\alpha^{2}=\alpha^{2} b-a \alpha^{3}\right)$.

Consequently we get a homomorphism $\varphi:(A,+) \rightarrow \operatorname{Pic}(A)$ by composing $A \rightarrow U(B / \mathfrak{f}), a \mapsto$ $\overline{1+a \alpha}$ and $j$.

Now assume that $A[\alpha]$ is reduced and replace $A$ by $A[X], B$ by $B[X]$ and $\alpha$ by $\alpha X$. We get a homomorphism $\varphi:(A[X],+) \rightarrow \operatorname{Pic}(A[X])$ which maps $P \in A[X]$ to the class of $M(X)=(1+$ $\alpha X P) A[X]+(\alpha X)^{2} A[X]$ (an $A[X]$-projective module). According to Proposition 2, this module is free if and only if there exists $V \in U(B[X])$ such that $V(1+\alpha X P) \in A[X]$. Since $B$ is reduced we 
have $U(B[X])=U(B)$. This gives the condition $\exists v \in U(B), v+\alpha v X P \in A[X]$, i.e., $v \in A$ and $\alpha v P \in A[X]$. Since $v$ is invertible in $B$ it is invertible in $A$ and the module $M(X)$ is free if and only if $\alpha P \in A[X]$. In case $P=1$ we retrieve "Schanuel's example", namely $M(0)$ is free, and $M(X)$ is extended (i.e., free) if and only if $\alpha \in A$. Concerning this topic see [7] pages 29-30 and 39-40.

\section{Reminder about dimension 1 (in constructive mathematics)}

One finds a constructive definition of Krull dimension in [2]. Applications of this notion have been given in $[3,4,6,8]$.

The trivial ring is characterized by dimension -1 . Concerning dimensions 0 and 1 , one has the following elementary characterizations.

Lemma 3 (dimensions 0 and 1) Let $A$ be be a commutative ring.

1. The ring $A$ is of dimension $\leq 0$ if and only if for all $x \in A$ there exist $n \in \mathbb{N}$ and $a \in A$ such that $x^{n}(1+a x)=0$.

2. The ring $A$ is of dimension $\leq 1$ if and only if for all $x, y \in A$ there exist $m, n \in \mathbb{N}$ and $a, b \in A$ such that $y^{m}\left(x^{n}(1+a x)+b y\right)=0$.

Moreover "usual rings" which are of dimension $\leq 0$ or 1 in classical mathematics satisfy in an explicit way the above elementary characterization.

The following lemma appears with a constructive proof in a slightly more general form in [6] (Lemma 4.3).

Lemma 4 (avoiding lemma in dimension 1) Let $A$ be an integral domain of dimension $\leq 1$. Let $\mathfrak{a}$ be an invertible ideal of $A$ and $\mathfrak{b}$ a nonzero ideal. Then there exists an element $u \neq 0$ of $\operatorname{Frac}(A)$ such that the ideal $u \mathfrak{a}$ is integral (i.e., contained in $A$ ) and comaximal to $\mathfrak{b}$.

The famous "one and a half theorem" is also constructively proven in [6] (Theorem 2.32) in the following general form.

Theorem 5 (one and a half theorem) Let $A$ be a commutative ring of dimension $\leq 1$ and $\mathfrak{a}$ an invertible ideal. Let $x \in \mathfrak{a}$ be a non-zerodivisor. Then there exists $y \in \mathfrak{a}$ such that for all $n \geq 1$, $\mathfrak{a}=x^{n} \mathfrak{a}+y A$. In particular, $\mathfrak{a}=\left\langle x^{n}, y\right\rangle$.

\section{An exact sequence for class groups}

\subsection{Context}

Let us consider two integral domains $A \subseteq B$ having the same fraction field.

The conductor c $(A, B)$ of $A$ in $B$ is the annihilator of the $A$-module $B / A$, and this is also the greatest ideal of $B$ contained in $A$.

We assume moreover that $A$ is a ring of dimension 1 and $B$ is a finitely generated $A$-module. So $B$ has dimension 1 .

The hypotheses "Frac $(A)=\operatorname{Frac}(B)$ " and " $B$ is a finitely generated $A$-module " imply that $B$ is a fractionary ideal of $A$ (consider a finite system of generators of $B$ over $A$ and multiply by a common $A$-denominator). In fact, $\operatorname{Frac}(A)=\operatorname{Frac}(B)$ says that $B / A$ is a torsion $A$-module and the fact that $B$ is a finitely generated $A$-module (equivalenty, $B / A$ is a finitely generated $A$-module) implies that its annihilator (i.e., the conductor of $A$ in $B$ ) is not reduced to 0 .

This context appears in algebraic number theory, namely $B$ is the ring of all integral numbers of a number field $K$ and $A$ is a number ring in $K$ (i.e., a subring of $B$ which is a $\mathbb{Z}$-module of rank equal to the dimension of $K$ as a $\mathbb{Q}$-vector space). 
There are other applications of this exact sequence. For example in geometry where rings $A$ and $B$ are finitely presented $\mathbf{k}[T]$-algebras (coordinate rings of affine curves). See [10] and the comment of J.-P. Serre in [12].

In the sequel we consider a nonzero ideal $\mathfrak{f}$ of $B$ contained in the conductor $\mathrm{c}(A, B)$.

\subsection{Back to the first exact sequence}

We get the following analogue of the first exact sequence (section 1.1):

$$
1 \longrightarrow U(A / \mathfrak{f}) \longrightarrow U(B / \mathfrak{f}) \stackrel{j}{\longrightarrow} \mathcal{I}_{\mathrm{fr}}(A) \stackrel{\pi}{\longrightarrow} \mathcal{I}_{\mathrm{fr}}(B)
$$

where $\pi$ is the canonical map $\mathfrak{a} \mapsto \mathfrak{a} B$ and $j$ is again defined by $\bar{b} \mapsto b A+\mathfrak{f}$. Proofs of the exactness up to $\mathcal{I}_{\mathrm{fr}}(A)$ are identical to that of section 1.1 and it remains to compute ker $\pi$. Let $\mathfrak{a}$ be a fractionary ideal of $A$ such that $\mathfrak{a} B=B$. Multiplying this equality by $\mathfrak{f}$, we get $\mathfrak{a} \mathfrak{f}=\mathfrak{f}$ and so $\mathfrak{f} \subseteq \mathfrak{a}$. By virtue of Theorem 5 , there exists $b \in \mathfrak{a}$ such that $b A+\mathfrak{f}=\mathfrak{a}$. We multiply this equality by $B$, we get $b B+\mathfrak{f}=\mathfrak{a} B=B, b \in B$ and $b$ is invertible modulo $\mathfrak{f}$, that is, $\mathfrak{a}=j(\bar{b})$.

This gives the following variant of Proposition 1.

Proposition 6 The map $j: U(B / \mathfrak{f}) \rightarrow \mathcal{I}_{\mathrm{fr}}(A)$ defined by $j(\bar{b})=b A+\mathfrak{f}$ induces an isomorphism from $U(B / \mathfrak{f}) / U(A / \mathfrak{f})$ onto the sub-group of $\mathcal{I}_{\mathrm{fr}}(A)$ made up of invertible fractionary ideals $\mathfrak{a}$ of $A$ such that $\mathfrak{a} B=B$.

\subsection{The canonical exact sequence}

The canonical exact sequence is the following analogue of the second exact sequence (section 1.2):

$$
1 \longrightarrow U(B) / U(A) \longrightarrow U(B / \mathfrak{f}) / U(A / \mathfrak{f}) \stackrel{j}{\longrightarrow} \operatorname{Pic}(A) \stackrel{\pi}{\longrightarrow} \operatorname{Pic}(B) \longrightarrow 1
$$

Since $B$ is an integral domain, the group $\operatorname{Pic}(B)$ is isomorphic to $\mathcal{I}_{\mathrm{fr}}(B) / \mathcal{P}_{\mathrm{fr}}(B)$. If $B$ is a Prüfer domain $\operatorname{Pic}(B)=\mathcal{I}_{\mathrm{fr}}(B) / \mathcal{P}_{\mathrm{fr}}(B)$ is the usual class group of finitely generated (fractionary) ideals.

Theorem 7 Let $A \subseteq B$ be two integral domains of dimension 1 with the same fraction field and such that $B$ is a finitely generated $A$-module. If $\mathfrak{f}$ is a nonzero ideal of $B$ contained in the conductor of $A$ in $B$, then we have the following exact sequence:

$$
1 \longrightarrow U(B) / U(A) \stackrel{i[b] \mapsto[b]}{\longrightarrow} U(B / \mathfrak{f}) / U(A / \mathfrak{f}) \stackrel{j[b] \mapsto[b A+\mathfrak{f}]}{\longrightarrow} \operatorname{Pic}(A) \stackrel{\pi[\mathfrak{a}] \mapsto[\mathfrak{a} B]}{\longrightarrow} \operatorname{Pic}(B) \longrightarrow 1
$$

\section{Proof.}

It remains to prove that $\pi$ is onto. Let $\mathfrak{b}$ be an invertible fractionary ideal of $B$. Applying the avoiding lemma in dimension 1 (Lemma 4), we can assume that $\mathfrak{b}$ is an integral ideal of $B$ comaximal to $\mathfrak{f}$. Let $1=b+f$ with $b \in \mathfrak{b}, f \in \mathfrak{f}$ and let us denote $\mathfrak{b}^{\prime}=b \mathfrak{b}^{-1}$. We have $b \in A$ because $\mathfrak{f} \subseteq A$. Moreover $\mathfrak{b}^{\prime}$ is an integral ideal of $B$ comaximal to $\mathfrak{f}$ (since $1=b+f \in \mathfrak{b}^{\prime}+\mathfrak{f}$ ) satisfying $\mathfrak{b} \mathfrak{b}^{\prime}=b B$. Let $\mathfrak{a}=\mathfrak{b} \cap A$ and $\mathfrak{a}^{\prime}=\mathfrak{b}^{\prime} \cap A$, Lemma 8 below implies that $\mathfrak{a} B=\mathfrak{b}$ and $\mathfrak{a}^{\prime}=b A$. So $\mathfrak{a}$ is invertible in $A$ and $\pi([\mathfrak{a}])=[\mathfrak{b}]$.

Lemma 8 (avoiding conductor lemma, Dedekind, cf. Sands [11] Theorem 3.1)

Let $A \subseteq B$ be two rings. Let $\mathfrak{f}$ be an ideal of $B$ contained in $\mathrm{c}(A, B)$. Then the two maps $\mathfrak{a} \mapsto \mathfrak{a} B$ and $\mathfrak{b} \mapsto \mathfrak{b} \cap A$ are reciprocal bijections one of the other. Moreover, they preserve intersection, sum and product when restricted to integral ideals comaximal to $\mathfrak{f}$. 


\section{Proof.}

We only show the first item. Let $\mathfrak{a}$ be an ideal of $A$ such that $\mathfrak{a}+\mathfrak{f}=A$. Then $\mathfrak{a} B \cap A=\mathfrak{a}$. Indeed, we have $a+f=1$ with $a \in \mathfrak{a}$ and $f \in \mathfrak{f}$. If $a^{\prime} \in \mathfrak{a} B \cap A$, then $a^{\prime}=a a^{\prime}+a^{\prime} f$ with $a a^{\prime} \in \mathfrak{a}$ and $a^{\prime} f \in \mathfrak{a} B f \subseteq \mathfrak{a} A=\mathfrak{a}$.

Let $\mathfrak{b}$ be an ideal of $B$ such that $f b+\mathfrak{f}=B$ and let us show that $(\mathfrak{b} \cap A) B=\mathfrak{b}$. We have $b+f=1$ with $b \in \mathfrak{b}$ and $f \in \mathfrak{f}$, thus $b \in \mathfrak{b} \cap A$. If $b^{\prime} \in \mathfrak{b}$, then $b^{\prime}=b b^{\prime}+b^{\prime} f$ with $b b^{\prime} \in(\mathfrak{b} \cap A) B$ and $b^{\prime} f \in \mathfrak{b} \cap A$.

\section{References}

[1] Bourbaki Algèbre commutative, chapitre 2, Localisation. Hermann 1961. 2

[2] Coquand T., Lombardi H. Hidden constructions in abstract algebra (3) Krull dimension of distributive lattices and commutative rings. in: Commutative ring theory and applications. Eds: Fontana M., Kabbaj S.-E., Wiegand S. Lecture notes in pure and applied mathematics vol 131. M. Dekker. (2002) 477-499. 2, 4

[3] Coquand T., Lombardi H., Quitté C. Generating non noetherian modules constructively. Manuscripta mathematica 115, (2004), 513-520. 2, 4

[4] Coquand T., Lombardi H., Quitté C. Dimension de Heitmann des treillis distributifs et des anneaux commutatifs Publications mathématiques de Besançon. Algèbre et Théorie des Nombres. (2006), 57-100. 2, 4

[5] Dedekind R. Über die Anzahl der Ideal-Klassen in den verschiedenen Ordnungen eines endlichen Körpers. 1-55 in Festschrift der Technischen Hochschule in Braunschweig zur Säkularfeier des Geburtstages von C. F. Gauß. 2

[6] Ducos L., Lombardi H., Quitté C., Salou M. Théorie algorithmique des anneaux arithmétiques, des anneaux de Prüfer et des anneaux de Dedekind. Journal of Algebra. 281, (2004), 604-650. 2, 4

[7] Lam T. Y. Lectures on Modules and Rings. Springer GTM 189 (1998). 4

[8] Lombardi H., Quitté C., Yengui I. Hidden constructions in abstract algebra (6) The theorem of Maroscia, Brewer and Costa. To appear in the Journal of Pure and Applied Algebra. 2, 4

[9] Mines R., Richman F., Ruitenburg W. A Course in Constructive Algebra. Springer-Verlag (1988). 2

[10] Rosenlicht M. Generalized Jacobian varieties. Ann. of Math. (2) 59 (1954), 505-530. 5

[11] Sands J. Generalization of a theorem of Siegel. Acta Arithmetica. 58 (1), (1991), 47-56. 1, 5

[12] Serre J.-P. Groupes algébriques et corps de classes. Hermann, Paris, 1975. 5

[13] Siegel C. Abschätzung von Einheiten. Nachr. Göttingen. 9, (1969), 71-86. 1

\section{Contents}

1 Exact sequences for groups of invertible modules 2

1.1 First exact sequence . . . . . . . . . . . . . . . . . . . . . 2

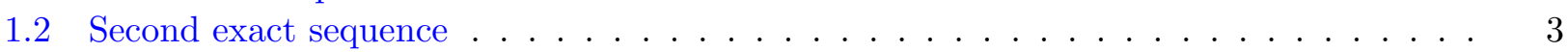

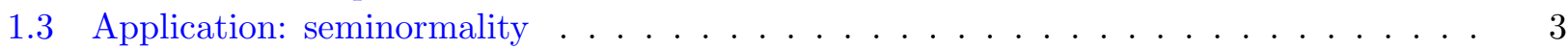

2 Reminder about dimension 1 (in constructive mathematics) 4

3 An exact sequence for class groups 4

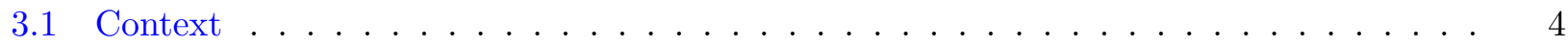

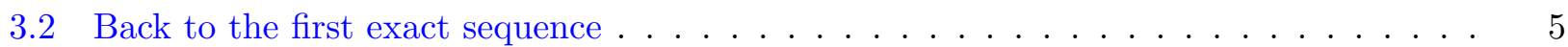

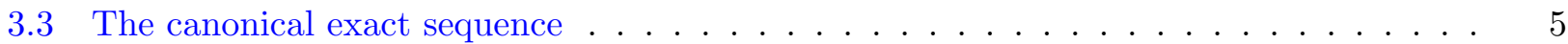

$\begin{array}{lr}\text { References } & 6\end{array}$ 